\title{
Bioinformatics profiling identifies 7 immune-related risk signatures for hepatocellular carcinoma
}

\author{
Feng Xue ${ }^{\text {Equal first author, } 1}$, Lixue Yang Equal first author, 1 , Binghua Dai ${ }^{2}$, Hui Xue ${ }^{1}$, Lei Zhang ${ }^{1}$, Ruiliang Ge ${ }^{\text {Corresp., } 1}$, Yanfu Sun \\ Corresp. 1 \\ ${ }^{1}$ Department of Hepatic Surgery II, Eastern Hepatobiliary Surgery Hospital, Shanghai, China \\ 2 Department of liver transplantation, Eastern Hepatobiliary Surgery Hospital, Shanghai, China \\ Corresponding Authors: Ruiliang Ge, Yanfu Sun \\ Email address: gerl_ehbh@sina.com, sunyanfu@hotmail.com
}

Background. Density of tumor infiltrating lymphocytes (TIL) and expressions of certain immune-related genes have prognostic and predictive values in hepatocellular carcinoma (HCC), however, factors determining the immunophenotype of HCC patients are still unclear. In the current study, the transcript sequencing data of liver cancer were systematically analyzed to determine an immune gene marker for the prediction of clinical outcome of HCC. Methods. RNASeq data and clinical follow-up information were downloaded from The Cancer Genome Atlas (TCGA), and the samples were assigned into high-stage and low-stage groups. Immune pathway-related genes were screened from the Molecular Signatures Database v4.0 (MsigDB) database. LASSO regression analysis was performed to identify robust immune-related biomarkers in predicting HCC clinical outcomes. Moreover, an immune gene-related prognostic model was established and validated by test sets and Gene Expression Omnibus (GEO) external validation sets. Results. We obtained 319 immune genes from MsigDB, and the genes have different expression profiles in high-stage and low-stage of HCC. Univariate survival analysis found that 17 genes had a significant effect on HCC prognosis, among them, 13 (76.5\%) genes were prognostically protective factors. Further lasso regression analysis identified 7 potential prognostic markers (IL27, CD1D, NCOA6, CTSE, FCGRT, CFHR1, and APOA2) of robustness, most of which are related to tumor development. Cox regression analysis was further performed to establish a 7 immune gene signature, which could stratify the risk of samples in training set, test set and external verification set $(p<0.01)$, and the AUC in both training set and test set was greater than 0.85 , which also greater compared with previous studies. Conclusion. This study constructed a 7-immunogenic marker as novel prognostic markers for predicting survival of HCC patients. 
1 Bioinformatics profiling identifies 7 immune-related risk signatures for hepatocellular 2 carcinoma

3 Feng Xue ${ }^{1, \mathrm{a}}$, Lixue Yang ${ }^{1, \mathrm{a}}$, Binghua Dai ${ }^{2}$, Hui Xue ${ }^{1}$, Lei Zhang ${ }^{1}$, Ruiliang Ge ${ }^{1, \mathrm{~b}}$, Yanfu Sun ${ }^{1, \mathrm{~b}}$

4 1. Department of Hepatic Surgery II, Eastern Hepatobiliary Surgery Hospital, No.225, Changhai

5 Rd, Shanghai, China

6 2. Department of liver transplantation, Eastern Hepatobiliary Surgery Hospital, No.225,

7 Changhai Rd, Shanghai, China

8 Corresponding Author:

9 Ruiliang Ge $\mathrm{e}^{1, \mathrm{~b}}$

10 No.225, Changhai Rd, Shanghai, 200438, China

11 Email address: gerl_ehbh@sina.com

12 Yanfu Sun ${ }^{1, b}$

13 No.225, Changhai Rd, Shanghai, 200438, China

14 Email address: sunyanfu@hotmail.com

15 a These authors contributed equally to this work.

b These authors contributed equally to this work. 


\section{Abstract}

Background. Density of tumor infiltrating lymphocytes (TIL) and expressions of certain immune-related genes have prognostic and predictive values in hepatocellular carcinoma (HCC), however, factors determining the immunophenotype of HCC patients are still unclear. $\square$ In the current study, the transcript sequencing data of liver cancer were systematically analyzed to determine an immune gene marker for the prediction of clinical outcome of HCC.

Methods. RNASeq data and clinical follow-up information were downloaded from The Cancer Genome Atlas (TCGA), and the samples were assigned into high-stage and low-stage groups. Immune pathway-related genes were screened from the Molecular Signatures Database v4.0 (MsigDB) database. LASSO regression analysis was performed to identify robust immunerelated biomarkers in predicting $\mathrm{HCC}$ clinical outcomes. Moreover, an immune gene-related prognostic model was established and validated by test sets and Gene Expression Omnibus (GEO) external validation sets.

Results. We obtained 319 immune genes from MsigDB, and the genes have different expression profiles in high-stage and low-stage of HCC. Univariate survival analysis found that 17 genes had a significant effect on HCC prognosis, among them, 13 (76.5\%) genes were prognostically protective factors. Further lasso regression analysis identified 7 potential prognostic markers (IL27, CD1D, NCOA6, CTSE, FCGRT, CFHR1, and APOA2) of robustness, most of which are related to tumor development. Cox regression analysis was further performed to establish a 7 immune gene signatures, which could stratify the risk of samples in training set, test set and external verification set $(\mathrm{p}<0.01)$, and the AUC in both training set and test set was greater than 0.85, which also greater compared with previous studies.

Conclusion. This study constructed a 7-immunogenic marker as novel prognostic markers for predicting survival of HCC patients.

1 Key words: Bioinformatics, Immunity, Prognostic Markers, TCGA

\section{Introduction}


53 As the most common malignant tumor in the liver, hepatocellular carcinoma (HCC) is the seventh most common cancer (Jemal et al. 2011) and the third leading cause of cancer-related deaths all over the world (Jemal et al. 2008). The recurrence rate of HCC within 5 years after surgery exceeds $70 \%$, causing HCC-related mortality of about 80\% (Bruix et al. 2005; Forner et al. 2012). The poor prognosis of patients with HCC is mainly resulted from the high progressiveness and metastasis of HCC and formation of new tumors in the diseased liver, which is the so-called "field effect" (Libbrecht et al. 2001; Sherman 2008). More than 70\% of HCC patients are unable to benefit from current treatments, due to potential liver dysfunction and advanced disease performance. The median survival time for patients with unresectable HCC is approximately 6 to 20 months, and their 5-year survival rate is less than 5\% (Mikhail et al. 2014). Therefore, it is highly urgent to discover prognostic biomarkers to accurately predict clinical outcomes of HCC patients.

Molecular profiles of tumor cells and cancer-related cells in their microenvironments are promising candidates for acting as predictive and prognostic biomarkers (Coussens \& Werb 2002; Gentles et al. 2015; Koscielny 2010; Tarhini et al. 2017; Topalian et al. 2012). Though significant advances have been made in high-throughput sequencing technology, clinical application of these technologies has been hampered by different tumor types andheterogeneity (Dalton \& Friend 2006; Dupuy \& Simon 2007; Koscielny 2010; Qi et al. 2016; Subramanian \& Simon 2010). Immune escape is a strategy, through which tumor cells evade host immune responses to continue their growth, and it is one of the hallmarks of human cancer (Corrales et al. 2017). Tumor immune escape is also considered as a promoter in the process of tumorigenesis and development, as it help tumor cells avoid or even block antigen stimulating immune response of tumor cells and provide appropriate microenvironment for tumor growth (Jiang et al. 2017; Kim et al. 2007). At present, studies have clarified the interaction between tumor and immune system (Angelova et al. 2015; Li et al. 2016; Rooney et al. 2015) and achieved remarkable progresses in advanced cancer treatment (Rooney et al. 2015), however, the treatment strategies only applies to a small number of patients.

Immunotherapy is another promising approach to overcome the poor prognosis after standard therapy as well as restricted applicability of targeted therapies to HCC patients. Currently, several immune-related parameters, mainly tumor infiltrating lymphocytes, have been reported to 
83 be able to predict the prognosis of HCC patients (Kim et al. 2018; Zhou et al. 2017), suggesting that immune states have a significant impact on the prognosis of HCC patients. Therefore, it is necessary to systematically study immunophenotype in HCC microenvironment, so as to better understand the complex anti-tumor response and help determine effective immunotherapy for HCC.

In the current study, expression profiles of HCC patients were obtained from large data sets from The Cancer Genome Atlas (TCGA) and Gene Expression Omnibus (GEO) databases, immune pathway genes were extracted from MsigDB, and different immune characteristics of $\mathrm{HCC}$ were analyzed through expression profiles of immune pathway genes, so as to investigate their performances in predicting survival of HCC patients and determine their clinical roles in existing staging models.

\section{Materials and Methods}

\section{Data collection and processing}

TCGA hepatocellular carcinoma RNA-Seq Counts data and clinical follow-up information concerning 306 tumor samples were downloaded from the HCCDB database (Lian et al. 2018) in November 5, 2018, finally 301 samples with stage information and followed up for more than one month were selected to be studied. Considering the age, gender, and stage distribution, we randomly divided the samples into two groups, namely, a training set $(\mathrm{N}=158)$ and a test set $(\mathrm{N}=143)$, as shown in Table1. Furthermore, total 319 genes related to immune system process M13664, immune response M19817, immune effector process M14818 and immune system development and the M3457 pathway were downloaded from MsigDB database. We performed $\log 2$ conversion on the Counts data and standardized the data using EBSeq (Leng et al. 2013) to obtain an expression profile. The GSE14520 (Roessler et al. 2010; Roessler et al. 2012) standardized expression profile data were obtained from the GEO database, and a total of 221 samples with follow-up information were screened as an external validation set.

\section{Analysis of immune characteristics between high-stage and low-stage}

In order to identify the difference in expressions of immune genes at different Stages, Stage I and II were seen as the Low Stage group, while Stage III and IV were seen as the high stage group, the gene expression differences between the two groups were analyzed using the R software 
112 package EBSeq (Leng et al. 2013). Further genetic Fold Change ranked as a row. GSEA

113 (Subramanian et al. 2007) was used for immunological pathway enrichment analysis, while t114 SNE30 (Li et al. 2017) was used to analyze the distribution of genes in the four immunological 115 pathways at high and low stages.

\section{Univariate Cox proportional hazards regression analysis}

As shown by Jin-Cheng et al (Guo et al. 2018), univariate Cox proportional-hazards regression analysis was performed on each of immunized genes to screen genes significantly associated with OS in the training data set. $\mathrm{P}<0.001$ was considered as the threshold.

\section{LASSO regression analysis}

LASSO is a widely used regression model with many potential prognostic features, as it can perform automatic feature selection that create signatures with generally good prognostic performance (Kostareli et al. 2016). LASSO method has been extended to Cox model for survival analysis, and has been successfully applied for the purpose of building sparse signatures for survival prognosis in many application areas, including oncology (Papaemmanuil et al. 2013; Yuan et al. 2014; Zhang et al. 2013). In the current study, the R package glmnet (Friedman et al. 2010) was used for robust prognostic feature screening of immune genes, and optimal features were evaluated by ten-fold cross-validation.

\section{Construction of prognostic immune gene signature}

To obtain robust prognostic feature of immune gene, we performed a multivariate Cox regression analysis and constructed the following risk scoring model:

$$
\text { RiskScore }=\sum_{k=1}^{n} \operatorname{Exp}_{k} * e_{k}^{H R}
$$

$\mathrm{N}$ refers to the number of prognostic immuno-genes, $\operatorname{Exp}_{k}$ is the expression value of prognostic immuno-genes, while $e_{k}^{H R}$ is estimated regression coefficient of immuno-genes in the multivariate Cox regression analysis.

\section{Correlation analysis between immune gene signature, GO and KEGG pathways}


137 R software package GSVA (Hanzelmann et al. 2013) was used for analyzing GO and KEGG 138 pathways of ssGSEA in each sample, and Pearson correlation between immune gene signature

139

140 and sample pathway enrichment score was further analyzed. Finally, GO Term and KEGG pathways that were the most related to immune gene signature were determined.

\section{RNA isolation and real-time PCR}

The HCC cells culture medium in each well was aspirated as much as possible. $1 \mathrm{ml}$ of Trizol (Invitrogen, Carlsbad, California) was added to the cells. The cells were placed horizontally for a while and blow evenly. The cells containing the lysate were transferred to a $1.5 \mathrm{ml}$ EP tube and kept at room temperature for 5 minutes. $200 \mu$ of chloroform was added to each tube and inverted for 15 seconds. After emulsification, let the tube stand for 5 minutes. After centrifugation at $12000 \mathrm{~g}$ at $4^{\circ} \mathrm{C}$ for 15 minutes, the upper aqueous phase was pipetted into a new $1.5 \mathrm{ml}$ of EP and an equal volume of isopropanol (about $400 \mu \mathrm{l}$ ) was added to each tube and allowed to keep at room temperature for $10 \mathrm{~min}$. After another centrifugation at $12,000 \mathrm{~g}$ at $4^{\circ} \mathrm{C}$ for 15 minutes, the supernatant was discarded and $1 \mathrm{ml}$ of pre-cooled $75 \%$ ice ethanol was added. After centrifugation at $7500 \mathrm{~g}$ at $4^{\circ} \mathrm{C}$ for 10 minutes, the supernatant was discarded. An appropriate amount of DEPC $(20 \mu \mathrm{l})$ was added to dissolve the RNA. The purity and concentration of RNA was tested by $260 \mathrm{~nm} / 280 \mathrm{~nm}$ using the NanoDrop nd-1000 spectrophotometer (NanoDrop Technologies, Wilmington, DE, USA). According to the program provided by the manufacturer (Thermo Fisher Scientific, Waltham, USA), reverse transcription cDNA kit was used to reverse transcribe 1 microgram total RNA for Synthesis of cDNA $\left(42^{\circ} \mathrm{C}\right.$ for 60 minutes, $70^{\circ} \mathrm{C}$ for 5 minutes, $4^{\circ} \mathrm{C}$ preservation). SYBR Green PCR Master Mix (Roche, Basle, Switzerland) was used to perform quantitative real-time polymerase chain reaction (RTqPCR) experiment using Opticon real-time PCR Detection System (ABI 7500, Life technology, USA). The PCR cycle was as follows: pretreatment at $95^{\circ} \mathrm{C}$ for 10 minutes; followed by 40 cycles of $94^{\circ} \mathrm{C}$ for 15 seconds, $60^{\circ} \mathrm{C}$ for 1 minutes, finally at $60^{\circ} \mathrm{C}$ for 1 minutes and at $4^{\circ} \mathrm{C}$ for preservation. The relative mRNA quantity was determined using the comparative cycle threshold $(\Delta \Delta \mathrm{Ct})$ method. GAPDH expression was used for normalization. The primer sequences were used for RT-qPCR analysis as Table S1.

\section{Statistical Analysis}


166 Kaplan-Meier (KM) curve was plotted, with the median risk score in each data set used as a cutoff in comparing the risk of survival between the high risk group (HC) and the low risk group (LC). Multivariate Cox regression analysis was performed to determine whether the immunegenic marker is an independent prognostic factor. Statistical significance was defined as $\mathrm{P}<0.05$. All analyses were performed in R 3.4.3, and all analyses were based on default parameters unless otherwise stated.

\section{Results}

\section{Different immunological characteristics exist in high stage and low stage}

Among the 301 samples obtained from TCGA, 220 cases of Stage I and II were found in the LC group, while 81 cases of Stage III and IV were in the HC group. The GESA analysis results showed that the LC group was in the immune response M19817, immune system process M13664, and immune effector process M14818 pathway activity in the LC group was higher than that in HC group (figure 1A, B, C). The expressions of 319 immune-related genes in all liver cancer samples was subjected to dimensionality reduction analysis using T-SNE, and the results demonstrated that the HC group was more concentrated compared with the LC group, indicating that these immune genes have different distribution characteristics (figure 1B). Thus, $\mathrm{HC}$ and LC had different immunological characteristics.

\section{Immune gene can be used as a prognostic marker}

Cox proportional hazard regression model (Kneale et al. 1981) was used for performing a univariate analysis of the relationship among 319 immune genes and HCC prognosis, as shown in Table 2, 17 genes were identified as significantly associated with prognosis, specifically, 4 genes were risk factors, while 13 genes were protective factors. Moreover, most of these genes were found related to complex diseases, for example, the most significant homozygous deletion of CFHR1 is associated with leukemia (Fratelli et al. 2016), APOA2 allelic imbalance is involved in endometrial cancer (Goumenou et al. 2001), and specific inhibition of SOD1 selectively promotes cancer cell apoptosis through regulating the ROS signaling network (Li et al. 2019). Furthermore, we performed a multivariate regression analysis to establish a risk model for 17 immune genes with the model's $\mathrm{AUC}=0.87$ (figure 2A). According to the proportion of the cases in $\mathrm{HC}$ and LC, 70\% quantile was taken as the threshold value of high-risk and low-risk 
195 grouping, that is, cutoff=-12.808. Significant prognostic differences between high and low group 196 samples were identified (Figure 2B). Similarly, in the test set AUC $=0.88$ (Figure 2C), the prognosis of the high-risk group was significantly worse than that of the low-risk group (Figure 2D). In conclusion, immune genes have great potential as prognostic markers.

\section{Identification of a 7-immu-gene signature for HCC survival}

Seventeen prognostically significant immune gene expression profiles were used in LASSO for dimensionality analysis in the training set, and 10-fold cross-validation was selected. When $\lambda=$ 0.02359167, the error rate was minimized (figure 3A), and 7 genes, namely, IL27, CD1D NCOA6, CTSE, FCGRT, CFHR1 and APOA2, were obtained, among which IL27 plays an important role in cancer and immunity (Fabbi et al. 2017), CD1D is a key factor in immunotherapy (King et al. 2018) and the homozygous deletion of CFHR1 is associated with acute myeloid leukemia (Fratelli et al. 2016). A 7-immuno-gene signature was established by a multivariate COX regression analysis with the model:

$$
\begin{aligned}
& \text { Risk }_{7} \\
& =-0.05983 * \text { CFHR1 }-0.01437 * \text { APOA2 - 0.10219* IL27 + } 0.10394 * \text { CTSE }-0.18521
\end{aligned}
$$

* FCGRT + $0.37195 *$ NCOA6 $-0.11573 *$ CD1D

The risk score of each sample were calculated, the samples were grouped according to $70 \%$ quantile of the risk score, and the relationship between the expressions of 7 genes and the risk scores were analyzed. We found that as protective factors, the low expressions of CFHR1, APOA2, IL27, FCGRT and CD1D were correlated with high risk of developing poor HCC prognosis, whereas high expressions of risk factors CTSE and NCOA6 were related to high risk of poor prognosis (figure $3 \mathrm{C}$ ). The prognosis was significantly different between the high-risk and low-risk groups (figure 4B), as in the training set, 7-gene signature had an AUC of 0.87 (figure 4A), while that the AUC in the TCGA test set was 0.89 (figure 4C). Moreover, the prognosis of high-risk samples was significantly worse than that of low-risk samples $(\mathrm{p}=0.0087$, figure 5D). Similarly, by applying the model to all TCGA samples, AUC=0.9 (figure 4E), and the prognosis of high-risk samples was significantly worse than that of low-risk samples $\mathrm{p}<0.0001$ (figure 4F). Consistent with the training set, the model was less effective in classifying prognosis result within the TCGA data set.

\section{Model performance evaluation}


223 The external dataset GSE14520 data was used for validating our model. The model was applied 224 to the GSE14520 data, and the risk score for each sample was calculated as AUC $=0.66$ (figure 225 5A). The samples are grouped into high and low risk groups according to their medians. The 226 prognosis in the high-risk group was found significantly worse than that in the low-risk group, with $\mathrm{p}<0.001$ (figure 5B), indicating that the model was applicable for risk stratification of data from different platforms. Furthermore, by analyzing a recently discovered 8-gene signature (Qiao et al. 2019), we compared the AUC in our model with that in the Guo et al. 's in the TCGA data for 1-, 3- and 5-year prognosis (figure 5C, D), and the results demonstrated that AUC in our model showed a better performance than the previous model.

\section{Immune gene signature, GO and KEGG pathway analysis}

Risk score of each sample was calculated based on the immune gene signature in the test sample, and the ssGSEA was used for analyzing GO and KEGG pathways. The Pearson correlation between the immune gene signature and the sample pathway enrichment score was also calculated. 5 GO term and KEGG Pathway with the highest correlation were selected for comparing them with the sample analysis scores (figure 6), and we observed a negative correlation between the PPAR signaling pathway and the sample risk value in the signaling pathway, while prion diseses, huntingtons disease, preroxisome and amyotrophic leteral sclerosis als pathway were positively correlated with sample risk. Moreover, in GO Term, all terms had a negative correlation with sample risk, including multiple immune system processes. To conclude, immune gene signatures are associated with pathways that are involved in a variety of complex diseases.

\section{The expression levels of genes in MHCC97}

We have demonstrated that 7 immune-related genes can be used as prognostic indicators for hepatocellular carcinoma, and further detected the expression of 7 genes on hepatocellular carcinoma MHCC97 cells by RT-qPCR assay. We found that the levels of CFHR1, APOA2, IL27, FCGRT and CD1D were significantly downregulated in MHCC97 cells, while the expressions of CTSE and NCOA6 were obviously upregulated in MHCC97 cells (figure 7). 
251 Those results indicated that those genes were associated with development of hepatocellular 252 carcinoma.

\section{Discussion}

254 In recent years, tumor immunotherapy has achieved remarkable success in the treatment of 255 advanced tumors (Maccio \& Madeddu 2012; Sharma et al. 2011). A comprehensive

256

257

258

259

260

261

262

263

264

265

266 understanding of liver cancer requires not only focus on tumor cells, but also on tumor microenvironment (Leonardi et al. 2012; Yarchoan et al. 2017), that contains a variety of cell populations interacting with cancer cells and different stages of tumorigenesis. The tumorinfiltrating immune cells and immune response in tumor microenvironment have attracted much research attention, and has become a promising therapeutic target. Further research into in what ways immune signatures are associated with HCC tumorigenesis and progression can contributes to the development of novel and specific targeted therapeutic strategies, especially in the setting of combined therapies. In the current study, the differences in immunological characteristics between the HC group and the LC group were analyzed, and we found that the immune response M19817, immune system process M13664, immune effector process and M14818 pathway were more active in LC group than that in the HC group in, suggesting that different tumor stages had different immune characteristics.

As HCC patients at similar TNM stages have different survival times, HCC is seen as a highly heterogeneous disease for its prognosis. As liver cancer is increasingly detected and treated in its early stages, traditional clinicopathological indicators such as tumor size, vascular invasion, portal vein tumor thrombus, and TNM staging could no longer satisfy the current needs in predicting individual outcomes. With the development of gene expression characteristics such as Oncotype DX (Siow et al. 2018) and Coloprint (Tan \& Tan 2011), it has been shown that gene expression profiling in screening new prognostic markers in cancers has become the most promising method for high-throughput molecular identification. Guo et al.(Qiao et al. 2019) have developed an 8-gene signature with an AUC around 0.7, however, the current study used immune gene set to construct 7- gene signature, with AUC reached 0.89, which suggested that our immune gene had higher predictive value as a prognostic marker. 
279 Our 7-immuno-gene signatures CFHR1, APOA2, IL27, FCGRT, and CD1D were protective factors, while CTSE and NCOA6 were risk factors. Urinary quantification of APOA2 protein was decscribed, which is a biomarker for the diagnosis of bladder cancer (Chen et al. 2015), and APOA2 had higher expression in liver tissues (Fukuhara et al. 2014), which differ from our study, consider cell heterogeneity. Shi L et al. found that the level of FCGRT was downregulation in clinical HCC samples (Shi et al. 2016). It has been reported that IL27 plays an important role in cancer and immunity (Fabbi et al. 2017), CD1D is a key factor in immunotherapy (King et al. 2018), and the homozygous deletion of CFHR1 is associated with acute myeloid leukemia (Fratelli et al. 2016), moreover, the stratified analysis of expression and prognosis found that NCOA6 is highly positively correlated with the grade of liver cancer, and this was the first time to prove that NCOA6 is associated with clinical stratification of liver cancer. Knockdown of signature gene CTSE for CD24+/CD44+ cells significantly reduced selfrenewal ability on HCC cells in vitro and in vivo (Ho et al. 2019). Meanwhile, our results of GSEA analysis indicated that the 7-immuno-gene signature-enriched pathway was significantly associated with tumorigenesis pathways and immune-related biological processes. These results indicate that the model developed in the current study has potential clinical application value, and can provide a potential target for the diagnosis of HCC patients.

Though bioinformatics techniques was applied to identify potential candidate immunogene markers involved in tumorigenesis in large samples, the current study still had certain limitations, for example, our samples lacked clinical follow-up information, thus, factors related to other health status of the HCC patients were not taken into investigation as prognostic biomarkers. Moreover, our results were obtained only through bioinformatics analysis, therefore, requires further experimental validations.

\section{Conclusion}

In conclusion, the current study systematically explored the immunogenic gene expression profiles in different aspects of liver cancer. We found 7-immuno-gene features in HCC, and had higher AUC value in both training and validation sets. Compared with clinical features, gene classifiers can improve survival risk prediction. Therefore, the classifier identified in the study could be used as a molecular diagnostic test in assessing the prognostic risk of HCC patients. 
None.

\section{References}

Angelova M, Charoentong P, Hackl H, Fischer ML, Snajder R, Krogsdam AM, Waldner MJ, Bindea G, Mlecnik B, Galon J, and Trajanoski Z. 2015. Characterization of the immunophenotypes and antigenomes of colorectal cancers reveals distinct tumor escape mechanisms and novel targets for immunotherapy. Genome Biol 16:64. 10.1186/s13059-015-0620-6

Bruix J, Sherman M, and Practice Guidelines Committee AAftSoLD. 2005. Management of hepatocellular carcinoma. Hepatology 42:1208-1236. 10.1002/hep.20933

Chen HC, Chen YT, Tsai RY, Chen MC, Chen SL, Xiao MC, Chen CL, and Hua MY. 2015. A sensitive and selective magnetic graphene composite-modified polycrystalline-silicon nanowire field-effect transistor for bladder cancer diagnosis. Biosens Bioelectron 66:198-207. 10.1016/j.bios.2014.11.019

Corrales L, Matson V, Flood B, Spranger S, and Gajewski TF. 2017. Innate immune signaling and regulation in cancer immunotherapy. Cell Res 27:96-108. 10.1038/cr.2016.149

Coussens LM, and Werb Z. 2002. Inflammation and cancer. Nature 420:860-867. 10.1038/nature01322

Dalton WS, and Friend SH. 2006. Cancer biomarkers--an invitation to the table. Science 312:1165-1168. $10.1126 /$ science. 1125948

Dupuy A, and Simon RM. 2007. Critical review of published microarray studies for cancer outcome and guidelines on statistical analysis and reporting. J Natl Cancer Inst 99:147-157. 10.1093/jnci/djk018

Fabbi M, Carbotti G, and Ferrini S. 2017. Dual Roles of IL-27 in Cancer Biology and Immunotherapy. Mediators Inflamm 2017:3958069. 10.1155/2017/3958069

Forner A, Llovet JM, and Bruix J. 2012. Hepatocellular carcinoma. Lancet 379:1245-1255. 10.1016/S01406736(11)61347-0

Fratelli M, Bolis M, Kurosaki M, Dori M, Guarnaccia V, Spinelli O, Alberti M, Valoti E, Pileggi S, Noris M, Remuzzi G, Rambaldi A, Terao M, and Garattini E. 2016. Association of CFHR1 homozygous deletion with acute myelogenous leukemia in the European population. Leuk Lymphoma 57:1234-1237. $10.3109 / 10428194.2015 .1082180$

Friedman J, Hastie T, and Tibshirani R. 2010. Regularization Paths for Generalized Linear Models via Coordinate Descent. J Stat Softw 33:1-22.

Fukuhara T, Wada M, Nakamura S, Ono C, Shiokawa M, Yamamoto S, Motomura T, Okamoto T, Okuzaki D, Yamamoto M, Saito I, Wakita T, Koike K, and Matsuura Y. 2014. Amphipathic alpha-helices in apolipoproteins are crucial to the formation of infectious hepatitis $\mathrm{C}$ virus particles. PLoS Pathog 10:e1004534. 10.1371/journal.ppat.1004534

Gentles AJ, Newman AM, Liu CL, Bratman SV, Feng W, Kim D, Nair VS, Xu Y, Khuong A, Hoang CD, Diehn M, West RB, Plevritis SK, and Alizadeh AA. 2015. The prognostic landscape of genes and infiltrating immune cells across human cancers. Nat Med 21:938-945. 10.1038/nm.3909

Goumenou AG, Arvanitis DA, Matalliotakis IM, Koumantakis EE, and Spandidos DA. 2001. Microsatellite DNA assays reveal an allelic imbalance in p16(Ink4), GALT, p53, and APOA2 loci in patients with endometriosis. Fertil Steril 75:160-165.

Guo JC, Wu Y, Chen Y, Pan F, Wu ZY, Zhang JS, Wu JY, Xu XE, Zhao JM, Li EM, Zhao Y, and Xu LY. 2018. Protein-coding genes combined with long noncoding RNA as a novel transcriptome molecular staging model to predict the survival of patients with esophageal squamous cell carcinoma. Cancer Commun (Lond) 38:4. 10.1186/s40880-018-0277-0

Hanzelmann S, Castelo R, and Guinney J. 2013. GSVA: gene set variation analysis for microarray and RNA-seq data. BMC Bioinformatics 14:7. 10.1186/1471-2105-14-7

Ho DW, Tsui YM, Sze KM, Chan LK, Cheung TT, Lee E, Sham PC, Tsui SK, Lee TK, and Ng IO. 2019. Singlecell transcriptomics reveals the landscape of intra-tumoral heterogeneity and stemness-related subpopulations in liver cancer. Cancer Lett 459:176-185. 10.1016/j.canlet.2019.06.002 
Jemal A, Bray F, Center MM, Ferlay J, Ward E, and Forman D. 2011. Global cancer statistics. CA Cancer J Clin 61:69-90. 10.3322/caac.20107

Jemal A, Siegel R, Ward E, Hao Y, Xu J, Murray T, and Thun MJ. 2008. Cancer statistics, 2008. CA Cancer J Clin 58:71-96. 10.3322/CA.2007.0010

Jiang D, Liu Y, Wang H, Wang H, Song Q, Sujie A, Huang J, Xu Y, Zeng H, Tan L, Hou Y, and Xu C. 2017. Tumour infiltrating lymphocytes correlate with improved survival in patients with esophageal squamous cell carcinoma. Sci Rep 7:44823. 10.1038/srep44823

Kim HD, Song GW, Park S, Jung MK, Kim MH, Kang HJ, Yoo C, Yi K, Kim KH, Eo S, Moon DB, Hong SM, Ju YS, Shin EC, Hwang S, and Park SH. 2018. Association Between Expression Level of PD1 by TumorInfiltrating CD8(+) T Cells and Features of Hepatocellular Carcinoma. Gastroenterology 155:1936-1950 e1917. 10.1053/j.gastro.2018.08.030

Kim R, Emi M, and Tanabe K. 2007. Cancer immunoediting from immune surveillance to immune escape. Immunology 121:1-14. 10.1111/j.1365-2567.2007.02587.x

King LA, Lameris R, de Gruij1 TD, and van der Vliet HJ. 2018. CD1d-Invariant Natural Killer T Cell-Based Cancer Immunotherapy: alpha-Galactosylceramide and Beyond. Front Immunol 9:1519. 10.3389/fimmu.2018.01519

Kneale GW, Mancuso TF, and Stewart AM. 1981. Hanford radiation study III: a cohort study of the cancer risks from radiation to workers at Hanford (1944-77 deaths) by the method of regression models in life-tables. $\mathrm{Br}$ $J$ Ind Med 38:156-166. 10.1136/oem.38.2.156

Koscielny S. 2010. Why most gene expression signatures of tumors have not been useful in the clinic. Sci Transl Med 2:14ps12. 10.1126/scitranslmed.3000313

Kostareli E, Hielscher T, Zucknick M, Baboci L, Wichmann G, Holzinger D, Mucke O, Pawlita M, Del Mistro A, Boscolo-Rizzo P, Da Mosto MC, Tirelli G, Plinkert P, Dietz A, Plass C, Weichenhan D, and Hess J. 2016. Gene promoter methylation signature predicts survival of head and neck squamous cell carcinoma patients. Epigenetics 11:61-73. 10.1080/15592294.2015.1137414

Leng N, Dawson JA, Thomson JA, Ruotti V, Rissman AI, Smits BM, Haag JD, Gould MN, Stewart RM, and Kendziorski C. 2013. EBSeq: an empirical Bayes hierarchical model for inference in RNA-seq experiments. Bioinformatics 29:1035-1043. 10.1093/bioinformatics/btt087

Leonardi GC, Candido S, Cervello M, Nicolosi D, Raiti F, Travali S, Spandidos DA, and Libra M. 2012. The tumor microenvironment in hepatocellular carcinoma (review). Int J Oncol 40:1733-1747. 10.3892/ijo.2012.1408

Li B, Severson E, Pignon JC, Zhao H, Li T, Novak J, Jiang P, Shen H, Aster JC, Rodig S, Signoretti S, Liu JS, and Liu XS. 2016. Comprehensive analyses of tumor immunity: implications for cancer immunotherapy. Genome Biol 17:174. 10.1186/s13059-016-1028-7

Li W, Cerise JE, Yang Y, and Han H. 2017. Application of t-SNE to human genetic data. J Bioinform Comput Biol 15:1750017. 10.1142/S0219720017500172

Li X, Chen Y, Zhao J, Shi J, Wang M, Qiu S, Hu Y, Xu Y, Cui Y, Liu C, and Liu C. 2019. The Specific Inhibition of SOD1 Selectively Promotes Apoptosis of Cancer Cells via Regulation of the ROS Signaling Network. Oxid Med Cell Longev 2019:9706792. 10.1155/2019/9706792

Lian Q, Wang S, Zhang G, Wang D, Luo G, Tang J, Chen L, and Gu J. 2018. HCCDB: A Database of Hepatocellular Carcinoma Expression Atlas. Genomics Proteomics Bioinformatics 16:269-275. 10.1016/j.gpb.2018.07.003

Libbrecht L, Craninx M, Nevens F, Desmet V, and Roskams T. 2001. Predictive value of liver cell dysplasia for development of hepatocellular carcinoma in patients with non-cirrhotic and cirrhotic chronic viral hepatitis. Histopathology 39:66-73.

Maccio A, and Madeddu C. 2012. Inflammation and ovarian cancer. Cytokine 58:133-147. 10.1016/j.cyto.2012.01.015

Mikhail S, Cosgrove D, and Zeidan A. 2014. Hepatocellular carcinoma: systemic therapies and future perspectives. Expert Rev Anticancer Ther 14:1205-1218. 10.1586/14737140.2014.949246

Papaemmanuil E, Gerstung M, Malcovati L, Tauro S, Gundem G, Van Loo P, Yoon CJ, Ellis P, Wedge DC, Pellagatti A, Shlien A, Groves MJ, Forbes SA, Raine K, Hinton J, Mudie LJ, McLaren S, Hardy C, Latimer C, Della Porta MG, O'Meara S, Ambaglio I, Galli A, Butler AP, Walldin G, Teague JW, Quek L, Sternberg A, Gambacorti-Passerini C, Cross NC, Green AR, Boultwood J, Vyas P, Hellstrom-Lindberg E, Bowen D, Cazzola M, Stratton MR, Campbell PJ, and Chronic Myeloid Disorders Working Group of the International Cancer Genome C. 2013. Clinical and biological implications of driver mutations in myelodysplastic syndromes. Blood 122:3616-3627; quiz 3699. 10.1182/blood-2013-08-518886 
Qi L, Chen L, Li Y, Qin Y, Pan R, Zhao W, Gu Y, Wang H, Wang R, Chen X, and Guo Z. 2016. Critical limitations of prognostic signatures based on risk scores summarized from gene expression levels: a case study for resected stage I non-small-cell lung cancer. Brief Bioinform 17:233-242. 10.1093/bib/bbv064

Qiao GJ, Chen L, Wu JC, and Li ZR. 2019. Identification of an eight-gene signature for survival prediction for patients with hepatocellular carcinoma based on integrated bioinformatics analysis. PeerJ 7:e6548. 10.7717 peerj.6548

Roessler S, Jia HL, Budhu A, Forgues M, Ye QH, Lee JS, Thorgeirsson SS, Sun Z, Tang ZY, Qin LX, and Wang XW. 2010. A unique metastasis gene signature enables prediction of tumor relapse in early-stage hepatocellular carcinoma patients. Cancer Res 70:10202-10212. 10.1158/0008-5472.CAN-10-2607

Roessler S, Long EL, Budhu A, Chen Y, Zhao X, Ji J, Walker R, Jia HL, Ye QH, Qin LX, Tang ZY, He P, Hunter KW, Thorgeirsson SS, Meltzer PS, and Wang XW. 2012. Integrative genomic identification of genes on $8 \mathrm{p}$ associated with hepatocellular carcinoma progression and patient survival. Gastroenterology 142:957-966 e912. 10.1053/j.gastro.2011.12.039

Rooney MS, Shukla SA, Wu CJ, Getz G, and Hacohen N. 2015. Molecular and genetic properties of tumors associated with local immune cytolytic activity. Cell 160:48-61. 10.1016/j.cell.2014.12.033

Sharma P, Wagner K, Wolchok JD, and Allison JP. 2011. Novel cancer immunotherapy agents with survival benefit: recent successes and next steps. Nat Rev Cancer 11:805-812. 10.1038/nrc3153

Sherman M. 2008. Recurrence of hepatocellular carcinoma. N Engl J Med 359:2045-2047. 10.1056/NEJMe0807581

Shi L, Zhang W, Zou F, Mei L, Wu G, and Teng Y. 2016. KLHL21, a novel gene that contributes to the progression of hepatocellular carcinoma. BMC Cancer 16:815. 10.1186/s12885-016-2851-7

Siow ZR, De Boer RH, Lindeman GJ, and Mann GB. 2018. Spotlight on the utility of the Oncotype DX((R)) breast cancer assay. Int $J$ Womens Health 10:89-100. 10.2147/IJWH.S124520

Subramanian A, Kuehn H, Gould J, Tamayo P, and Mesirov JP. 2007. GSEA-P: a desktop application for Gene Set Enrichment Analysis. Bioinformatics 23:3251-3253. 10.1093/bioinformatics/btm369

Subramanian J, and Simon R. 2010. Gene expression-based prognostic signatures in lung cancer: ready for clinical use? J Natl Cancer Inst 102:464-474. 10.1093/jnci/djq025

Tan IB, and Tan P. 2011. Genetics: an 18-gene signature (ColoPrint(R)) for colon cancer prognosis. Nat Rev Clin Oncol 8:131-133. 10.1038/nrclinonc.2010.229

Tarhini AA, Lin Y, Lin HM, Vallabhaneni P, Sander C, LaFramboise W, and Hamieh L. 2017. Expression profiles of immune-related genes are associated with neoadjuvant ipilimumab clinical benefit. Oncoimmunology 6:e1231291. 10.1080/2162402X.2016.1231291

Topalian SL, Hodi FS, Brahmer JR, Gettinger SN, Smith DC, McDermott DF, Powderly JD, Carvajal RD, Sosman JA, Atkins MB, Leming PD, Spigel DR, Antonia SJ, Horn L, Drake CG, Pardoll DM, Chen L, Sharfman WH, Anders RA, Taube JM, McMiller TL, Xu H, Korman AJ, Jure-Kunkel M, Agrawal S, McDonald D, Kollia GD, Gupta A, Wigginton JM, and Sznol M. 2012. Safety, activity, and immune correlates of antiPD-1 antibody in cancer. N Engl J Med 366:2443-2454. 10.1056/NEJMoa1200690

Yarchoan M, Xing D, Luan L, Xu H, Sharma RB, Popovic A, Pawlik TM, Kim AK, Zhu Q, Jaffee EM, Taube JM, and Anders RA. 2017. Characterization of the Immune Microenvironment in Hepatocellular Carcinoma. Clin Cancer Res 23:7333-7339. 10.1158/1078-0432.CCR-17-0950

Yuan Y, Van Allen EM, Omberg L, Wagle N, Amin-Mansour A, Sokolov A, Byers LA, Xu Y, Hess KR, Diao L, Han L, Huang X, Lawrence MS, Weinstein JN, Stuart JM, Mills GB, Garraway LA, Margolin AA, Getz G, and Liang H. 2014. Assessing the clinical utility of cancer genomic and proteomic data across tumor types. Nat Biotechnol 32:644-652. 10.1038/nbt.2940

Zhang JX, Song W, Chen ZH, Wei JH, Liao YJ, Lei J, Hu M, Chen GZ, Liao B, Lu J, Zhao HW, Chen W, He YL, Wang HY, Xie D, and Luo JH. 2013. Prognostic and predictive value of a microRNA signature in stage II colon cancer: a microRNA expression analysis. Lancet Oncol 14:1295-1306. 10.1016/S14702045(13)70491-1

Zhou G, Sprengers D, Boor PPC, Doukas M, Schutz H, Mancham S, Pedroza-Gonzalez A, Polak WG, de Jonge J, Gaspersz M, Dong H, Thielemans K, Pan Q, JNM IJ, Bruno MJ, and Kwekkeboom J. 2017. Antibodies Against Immune Checkpoint Molecules Restore Functions of Tumor-Infiltrating T Cells in Hepatocellular Carcinomas. Gastroenterology 153:1107-1119 e1110. 10.1053/j.gastro.2017.06.017 


\section{Table $\mathbf{1}$ (on next page)}

Random grouping information of TCGA samples DAQAB18. 
1 Table 1: Random grouping information of TCGA samples

\begin{tabular}{cccc}
\hline & & Traning set & Test set \\
\hline \multirow{2}{*}{ age } & $<=60$ & 80 & 73 \\
& $>60$ & 78 & 70 \\
\cline { 2 - 4 } Gender & Male & 110 & 96 \\
& Female & 48 & 47 \\
\cline { 2 - 4 } Stage & High & 41 & 40 \\
& Low & 117 & 103 \\
\cline { 2 - 4 } & & 158 & 143
\end{tabular}

2

3 
Table 2 (on next page)

17 prognostic immune genes 
1 Table 2. 17 prognostic immune genes

\begin{tabular}{|c|c|c|c|c|c|}
\hline Genes & SYMBOL & $\ln H R$ & $\begin{array}{l}\text { HR } \\
\text { (95\%_CI_for_HR) }\end{array}$ & wald.test & p.value \\
\hline 3078 & CFHR1 & -0.26 & $0.77(0.69-0.85)$ & 25 & $6.90 \mathrm{E}-07$ \\
\hline 336 & APOA2 & -0.24 & $0.79(0.72-0.87)$ & 24 & $9.60 \mathrm{E}-07$ \\
\hline 717 & $\mathrm{C} 2$ & -0.49 & $0.61(0.5-0.75)$ & 24 & $1.20 \mathrm{E}-06$ \\
\hline 6647 & SOD1 & -0.93 & $0.4(0.27-0.58)$ & 22 & $3.30 \mathrm{E}-06$ \\
\hline 7448 & VTN & -0.28 & $0.75(0.67-0.85)$ & 21 & $4.40 \mathrm{E}-06$ \\
\hline 7494 & XBP1 & -0.94 & $0.39(0.25-0.6)$ & 18 & $2.30 \mathrm{E}-05$ \\
\hline 6288 & SAA1 & -0.19 & $0.83(0.76-0.91)$ & 17 & $3.70 \mathrm{E}-05$ \\
\hline 1394 & CRHR1 & 0.62 & $1.9(1.4-2.5)$ & 15 & $8.80 \mathrm{E}-05$ \\
\hline 335 & APOA1 & -0.18 & $0.83(0.76-0.91)$ & 15 & 0.00011 \\
\hline 6480 & ST6GAL1 & -0.45 & $0.64(0.5-0.8)$ & 14 & 0.00014 \\
\hline 246778 & IL27 & -0.32 & $0.72(0.61-0.86)$ & 13 & 0.00031 \\
\hline 1510 & CTSE & 0.18 & $1.2(1.1-1.3)$ & 12 & 0.00044 \\
\hline 2217 & FCGRT & -0.77 & $0.46(0.3-0.72)$ & 12 & 0.00064 \\
\hline 23054 & NCOA6 & 1 & $2.8(1.5-5.1)$ & 11 & 0.00073 \\
\hline 85477 & SCIN & 0.24 & $1.3(1.1-1.5)$ & 11 & 0.00079 \\
\hline 7704 & ZBTB16 & -0.27 & $0.76(0.65-0.89)$ & 11 & 0.00081 \\
\hline 912 & CD1D & -0.3 & $0.74(0.62-0.89)$ & 11 & 0.001 \\
\hline
\end{tabular}

2 


\section{Figure 1}

Different immunological characteristics exist in high stage and low stage

A-C: GSEA enrichment for immune response, immune system process and immune effector process pathway, na-pos means HC group, na-neg means LC group, three pathways for FDR $<0.001$. D: The expression of 319 immune-related genes were conducted to Dimensional analysis of T-SNE. Red indicates the LC group and black indicates the HC group. 
A

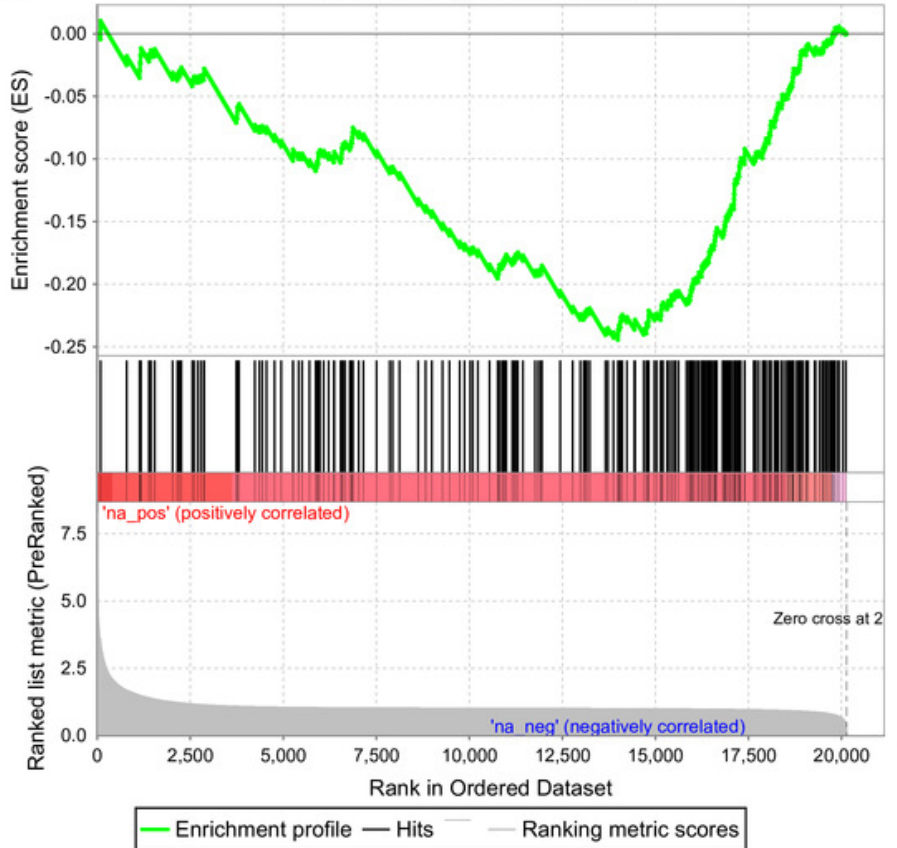

C

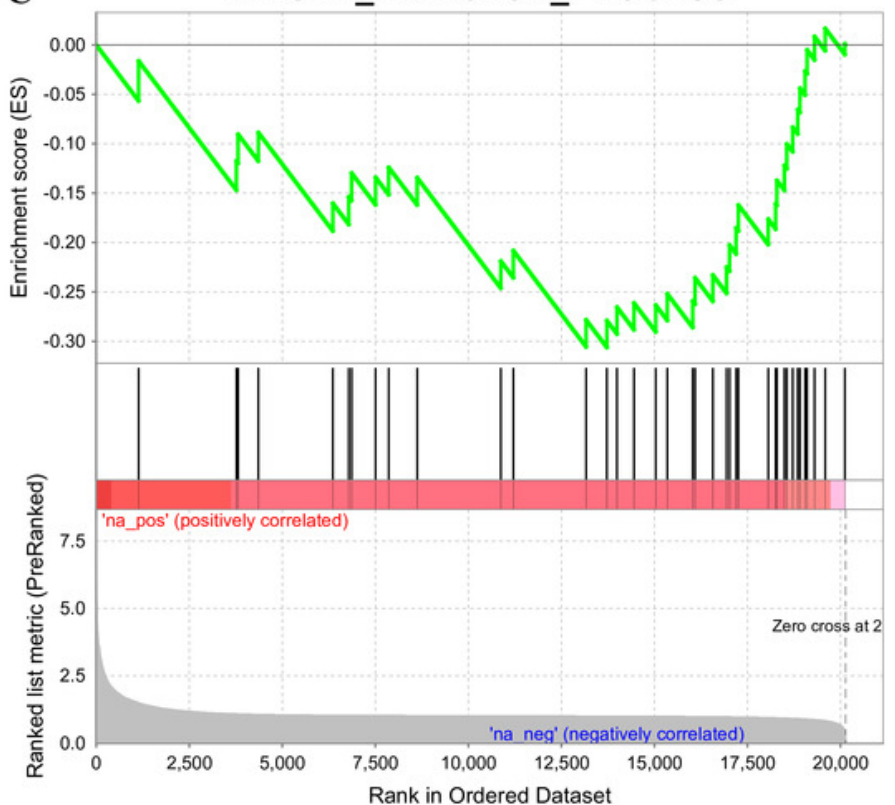

- Enrichment profile — Hits

Ranking metric scores
B

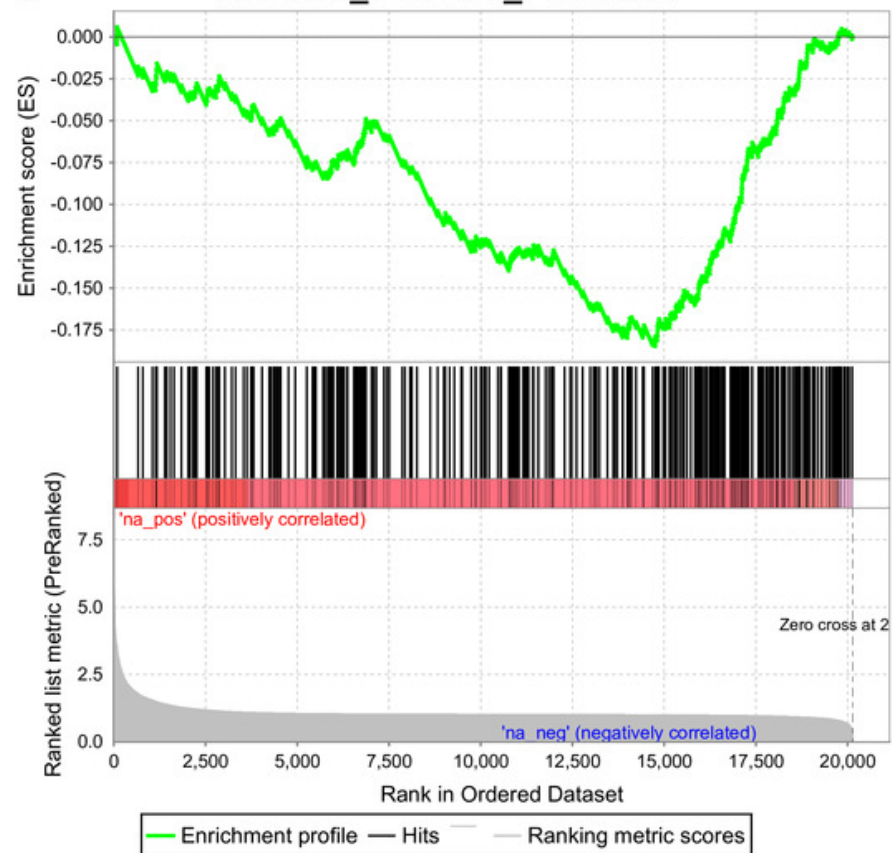

D

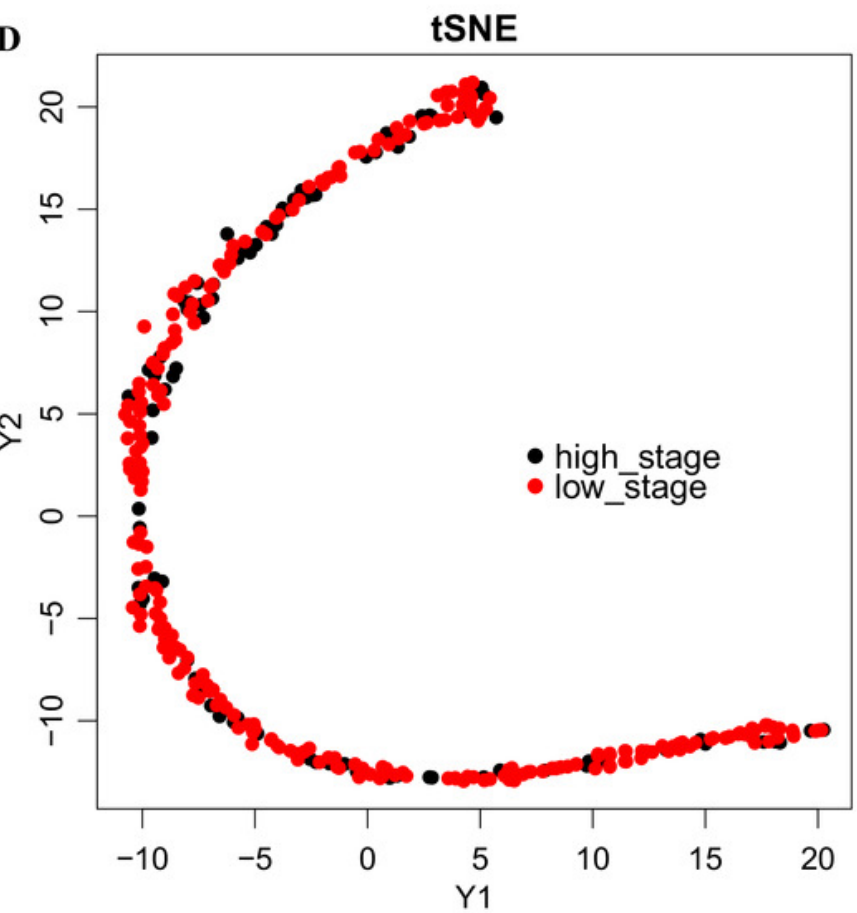


Figure 2

Immune gene can be used as a prognostic marker ps://

A: AUC curve of 17 immune genes in the TCGA training set. B: Kaplan-meier curve of prognosis difference between high and low risk group of TCGA training group. C: AUC curve of 17 immune genes in TCGA test set. D: Kaplan-meier curve of prognosis difference between high and low risk group of TCGA test group. 
A

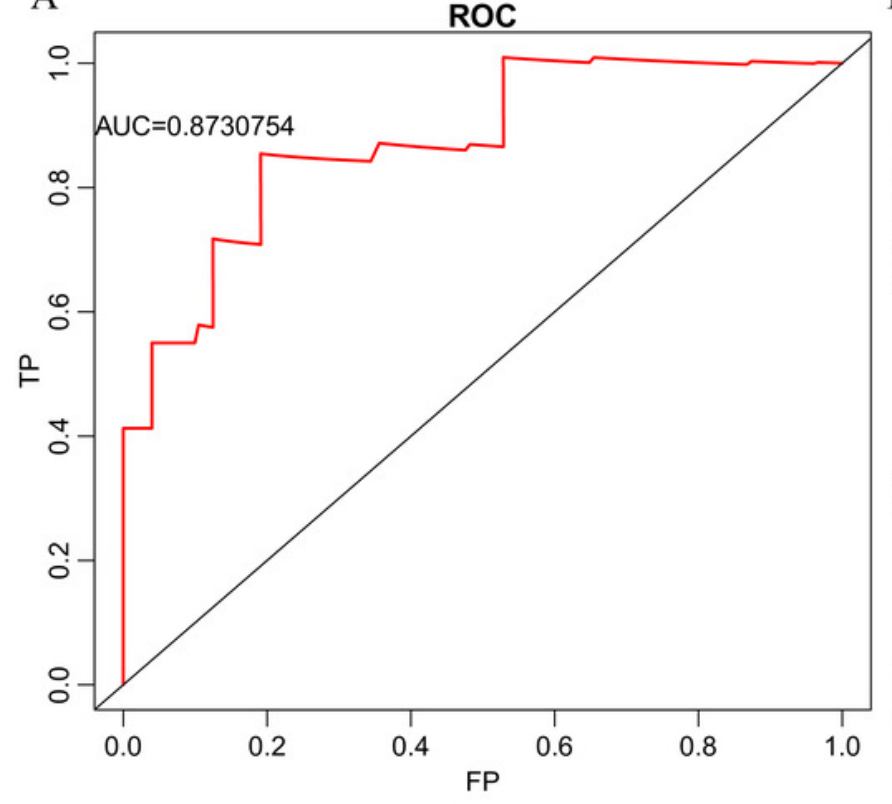

C

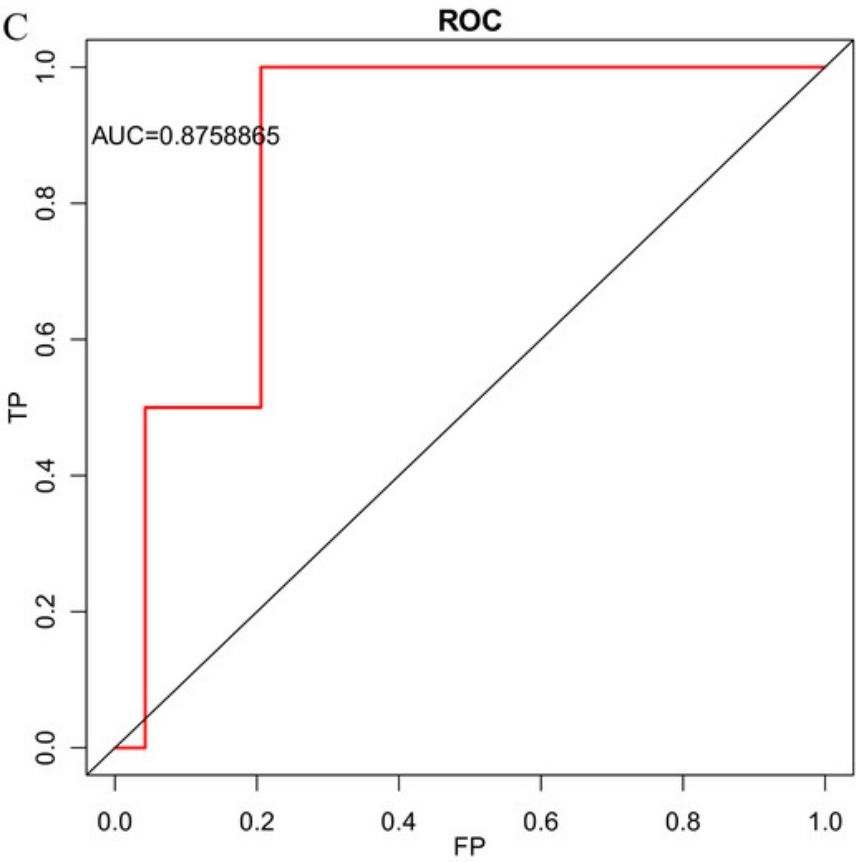

B

Strata - risk=High - risk=Low
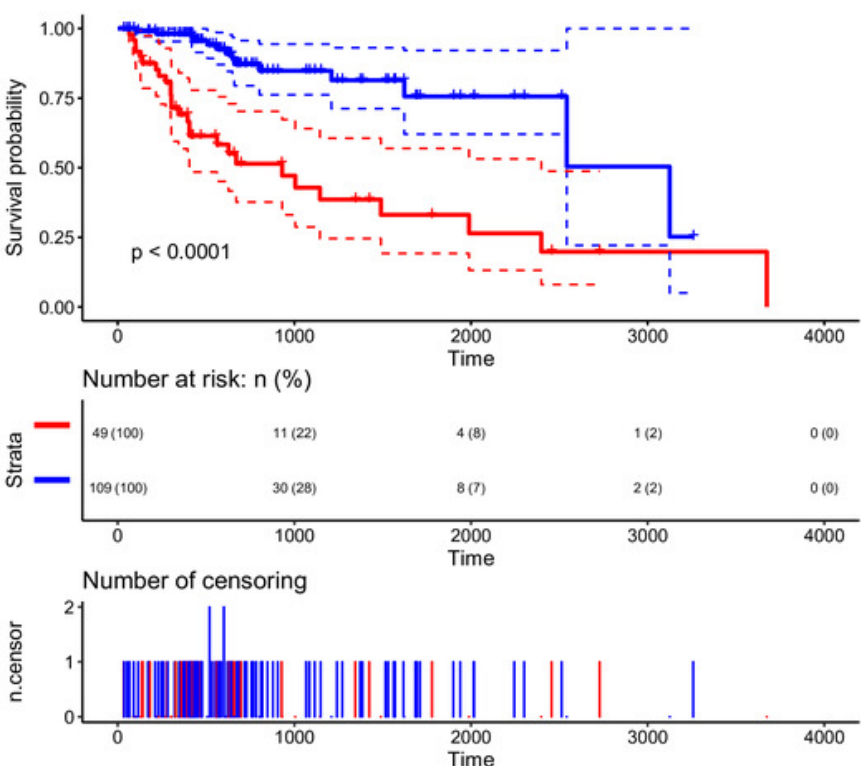

$\mathrm{D}$

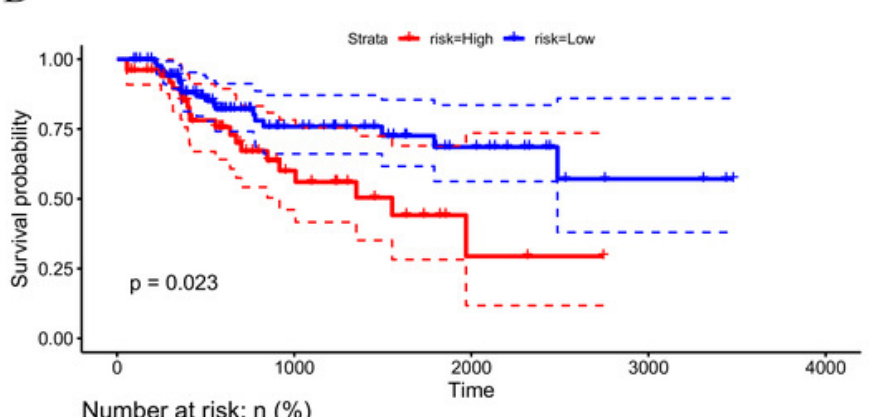

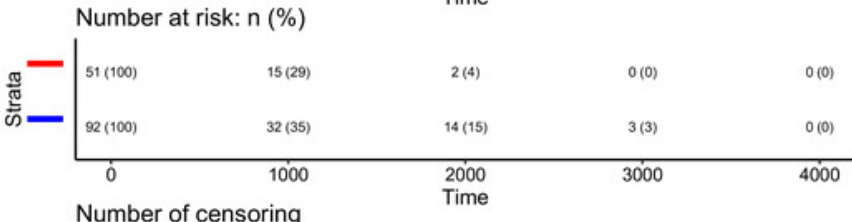

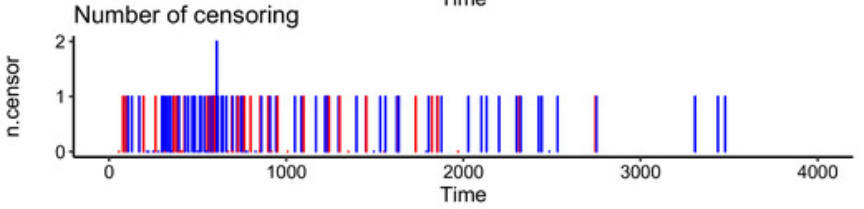


Figure 3

Identification of a 7-immu-gene signature for HCC survival

Lasso regression analysis results: A: the trajectory of each independent variable, the horizontal axis represents the log value of the independent lambda, the vertical axis represents the coefficient of the independent variable. B: the average error rate interval under each lambda. C: The relationship between 7 immune genes and risk scores.
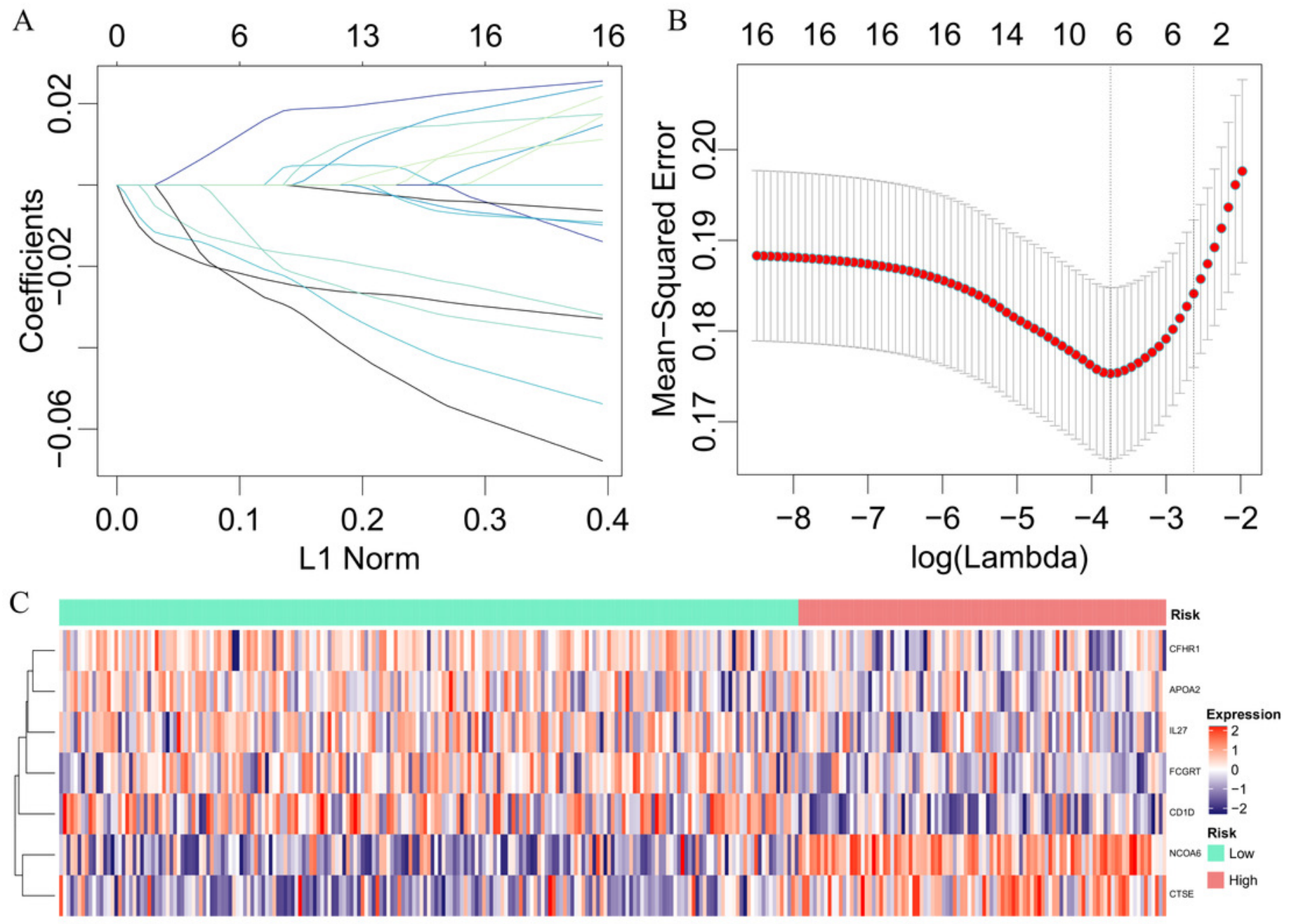
Figure 4

Identification of a 7-immu-gene signature for HCC survival 5

A: ROC curve of TCGA training set. B: Kaplan-meier curve of high and low risk group prognosis difference in TCGA training set. C: ROC curve of TCGA test set. D: Kaplan-meier curve of high and low risk group prognosis difference in TCGA test set. E: ROC curve of the dataset of all samples of TCGA. F: Kaplan-meier curve of prognosis difference between high and low risk groups in the dataset of all samples of TCGA.
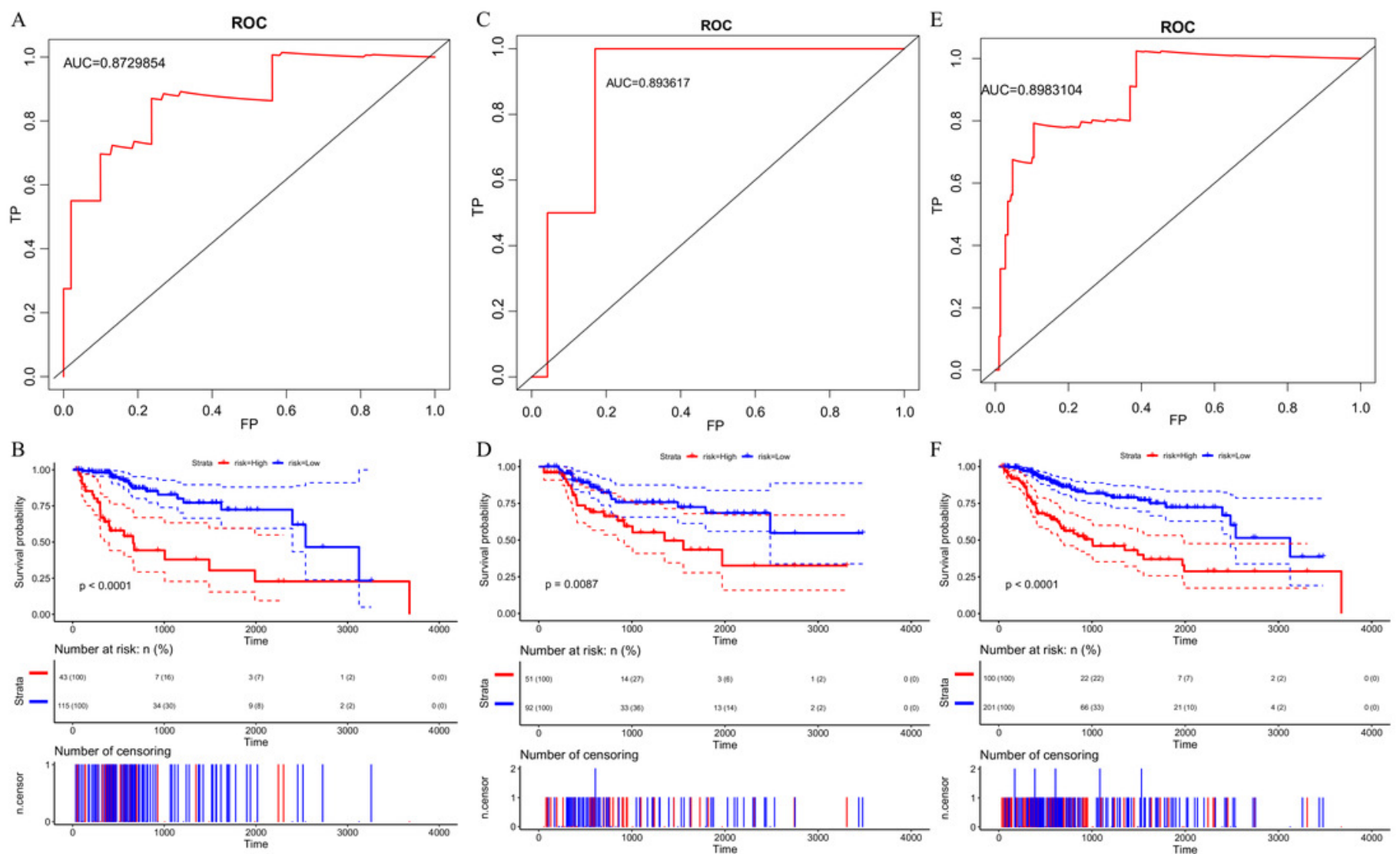
Figure 5

Model performance evaluation ashes

A: ROC curve of the external verification data set. B: Kaplan-meier curve for prognosis differences between high and low risk groups in the external validation data set. C: ROC curve of the model in the TCGA data set for 1 year, 3 years, and 5 years. D: AUC of the Guo et al. model in the TCGA data for 1 year, 3 years and 5 years. 

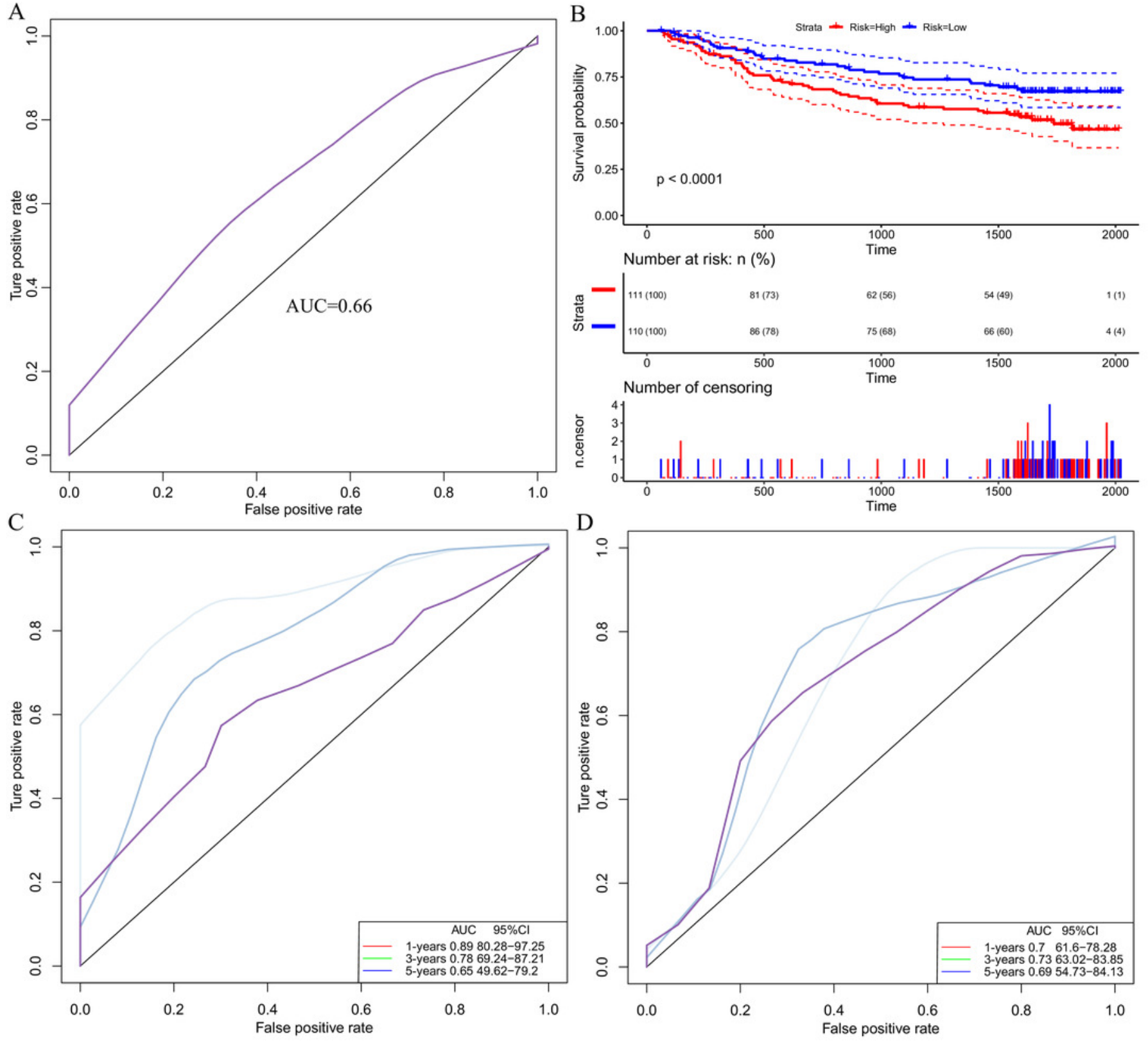
Figure 6

Immune gene signature, GO and KEGG pathway analysis

Immune gene signature and GO and KEGG pathway analysis, horizontal representation of samples, heat map representation of GO, KEGG pathway enrichment scores.

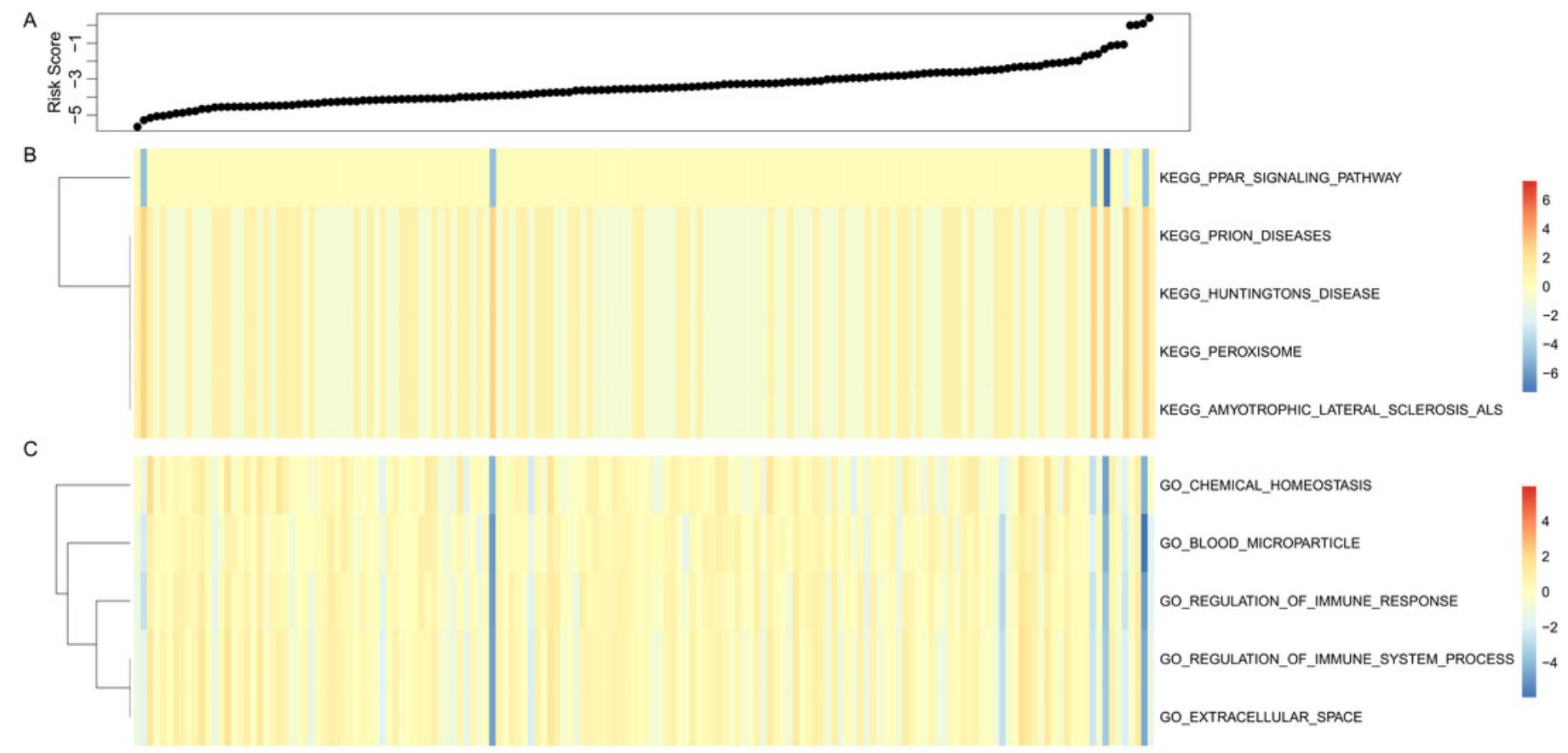


Figure 7

The expressions of genes in MHCC97.

The levels of CFHR1, APOA2, IL27, FCGRT and CD1D were significantly downregulated in MHCC97 cells, while the expressions of CTSE and NCOA6 were obviously upregulated in MHCC97 cells.

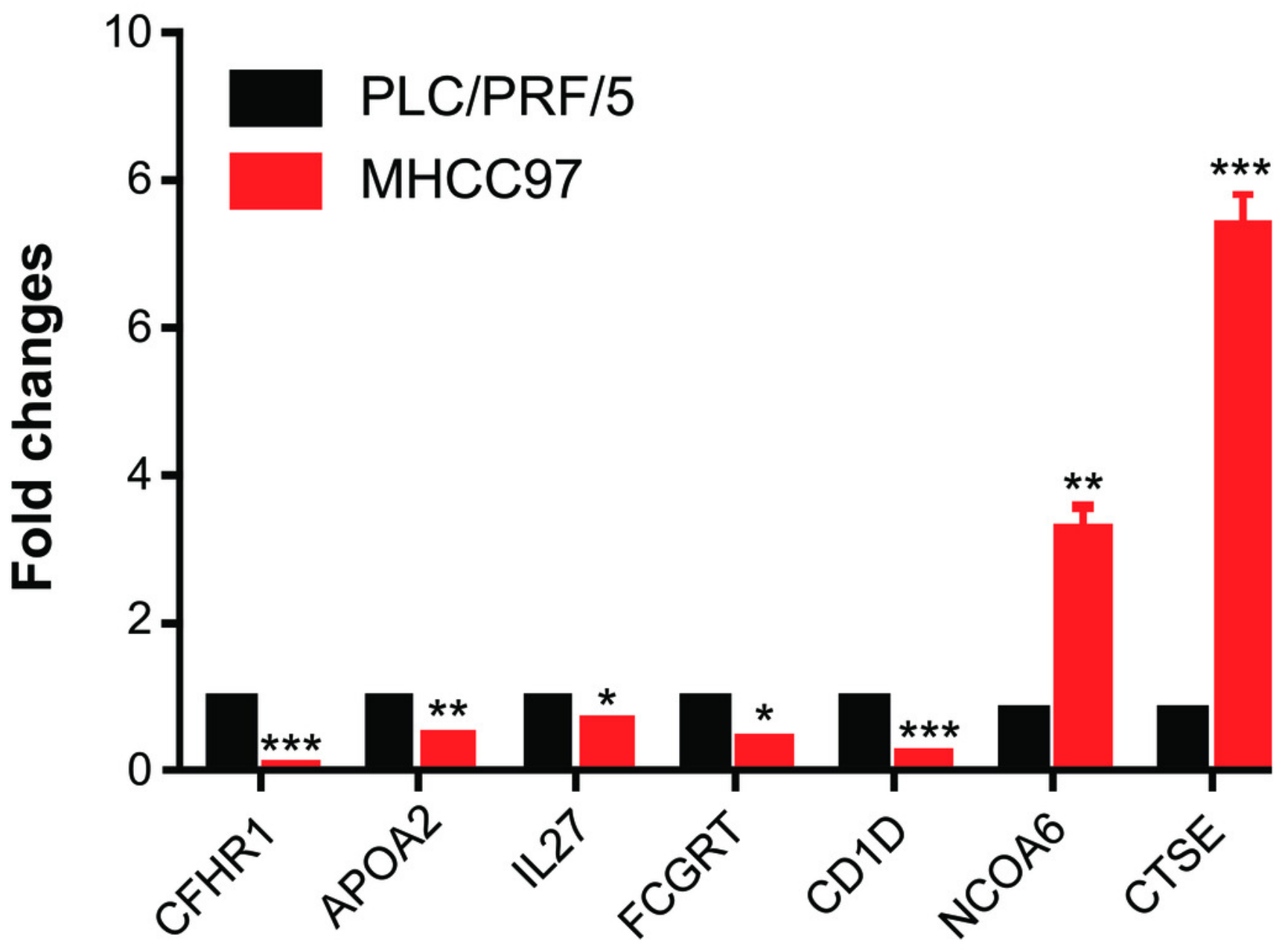

